\title{
A modified single-armed technique for microsurgical vasoepididymostomy
}

\author{
Liang Zhao ${ }^{1, *}$, Chun-Hua Deng ${ }^{2, *}$, Xiang-Zhou Sun ${ }^{2}$, Yu Chen ${ }^{1}$, Wen-Wei Wang ${ }^{1}$, Liang-Yun Zhao ${ }^{1}$, \\ Ling-You Zeng ${ }^{1}$ and Xiang-An Tu ${ }^{1}$
}

This study is to evaluate the effectiveness of a modified single-armed suture technique for microsurgical vasoepididymostomy (VE) in patients with epididymal obstructive azoospermia. From September 2011 to December 2011, microsurgical two-suture longitudinal intussusception VEs were performed using our modified single-armed suture technique in 17 men with epididymal obstructive azoospermia at our hospital. Two of these patients underwent repeated VEs after previous failed VEs, and one patient underwent unilateral VE because of an occlusion of the left abdominal vas deferens. The presence of sperm in the semen sample at 3 months postoperation was used as the preliminary endpoint of this study. Each patient provided at least one semen sample at the 3-month time point, and the patency was assessed by the reappearance of sperm $\left(>10^{4} \mathrm{ml}^{-1}\right)$ in the semen. The mean operative time for the modified technique was $219 \mathrm{~min}$. Patency was noted in 10 men (58.8\%), including one patient who underwent repeated VE. The patient who underwent unilateral anastomosis manifested no sperm postoperatively in his semen. Sperm granulomas were not detected in this cohort. The results of this study demonstrate that our modified technique for microsurgical longitudinal intussusception VE is effective. We believe that it is a practical alternative that may reduce operation time and obviate the suture crossing.

Asian Journal of Andrology (2013) 15, 79-82; doi:10.1038/aja.2012.100; published online 8 October 2012

Keywords: epididymis; microsurgery; obstructive azoospermia; single-armed technique; vasoepididymostomy (VE)

\section{INTRODUCTION}

Microsurgical reconstruction of the male reproductive tract has been demonstrated to be more cost-effective than assisted reproductive technologies such as in vitro fertilisation and intracytoplasmic sperm injection. ${ }^{1,2}$ In cases of epididymal obstruction, an intussusception vasoepididymostomy (VE) is required to reestablish the continuity of the sperm transportation pathway. However, intussusception VE is known as one of the most technically challenging microsurgical procedures because the patency rate is dependent on the surgeon's microsurgical experience and technique when performing such a small anastomosis between the vas deferens and delicate epididymal tubules. ${ }^{3}$ Based on Berger's three-suture triangulation ${ }^{4}$ and Marmar's two-suture transverse anastomotic techniques, ${ }^{5}$ Chan et al. ${ }^{6}$ described a two-suture longitudinal intussusception VE (LIVE) technique in a rat model, and the postoperative results indicated a superior patency rate and a lower rate of granuloma formation. In these studies, ${ }^{4-6}$ two expensive specialized double-armed, 10-0 nylon sutures, which are scarce in most countries, were used to avoid back-walling the vas deferens. In 2007, Monoski et al. ${ }^{7}$ used a novel single-armed suture technique for the LIVE technique in a rat model and resolved the difficulty in obtaining the specialized double-armed sutures. In this study, we describe a modified single-armed suture LIVE technique in patients with epididymal obstructive azoospermia (EOA). To our knowledge, this is the first study in humans to use a single-armed suture LIVE technique to treat epididymal obstruction.

\section{MATERIALS AND METHODS}

Patients

Our diagnostic criteria for the inclusion of patients in this study, briefly described here, are as follows: ${ }^{8}$ azoospermia was confirmed by two routine semen assays over an interval of at least 6 weeks; normal semen volume, $\mathrm{pH}$ value and seminal plasma fructose were confirmed by at least two semen samples; the patients' karyotypes were analysed preoperatively and no euchromosome or sex chromosome abnormalities were detected; follicle-stimulating hormone levels were measured and found to be within normal limits; scrotal and rectal ultrasonography showed normal testis volume without dilation of the ejaculatory duct or seminal vesicle; and the subjects' female partners underwent evaluation by a gynaecologist and were reported to be fertile.

From September 2011 to December 2011, 17 patients with an average age of 30.4 years were diagnosed with EOA at our hospital. The average duration of sterility was 38.4 (12-96) months.

\section{VE approaches}

A Leica M520 MC-1 operating microscope (Leica Microsystems (Schweiz) AG, Heerbrugg, Switzerland) was used to perform the 
microsurgical procedure at a magnification of 7-12. After vasal hemisection, distal patency was confirmed by infusing diluted methylene blue through the vas deferens, resulting in blue colouring of the urine. A suitable site for anastomosis on the epididymis was identified by examining the tubules under the microscope for dilatation. The epididymal adventitia was incised roundly, and the vas deferens was anchored to it with two 8-0 prolene sutures through the vasal seromuscular layer. Our modified single-armed suture LIVE technique was performed using two of the same single-armed, 10-0 prolene sutures (Ethicon W2790 (length $3.8 \mathrm{~mm}, 200 \mu \mathrm{m}$ and curvature 3/ $8)$ ) with a trimmed length of $4-5 \mathrm{~cm}$. The first suture was placed in an outside-in fashion through the mucosal layer of the vas deferens at point al (Figure 1). Then, the needle was used to pierce the lateral aspect of the epididymal tubule and was placed longitudinally. The second 10-0 single-armed prolene suture was placed identically through point b1 on the vas deferens, parallel to the first suture on the contralateral side of the epididymal tubule. The placement of these two sutures was a modification of the technique described by Monoski et al., ${ }^{7}$ who placed vasa deferentia sutures at points a2 and b2 in an outside-in fashion. This minor modification resulted in a low position of the two sutures when the needles were in the tubule, which, in our experience, could avoid crossing of the sutures and reduce operation times.

After opening the tubule between the two parallel needles and examining the exuded epididymal fluid for sperm, the needles were pulled out and placed inside-out through the mucosal layer of the vas deferens at points $\mathrm{a} 2$ and $\mathrm{b} 2$. The sutures were tied down, and then, the epididymal tunic was secured to the vasal muscle and adventitia with 8-10 interrupted 8-0 prolene stitches. If sperm were not found in epididymal fluid, the procedure was repeated at a closer segment of the epididymis. The VE was not continued if repeated examinations failed to manifest sperm in the epididymal fluid or patency was not apparent following assessment of the abdominal side of the vas deferens.

Sexual abstinence was advised for 6 weeks after surgery. Chinese patent medicines were introduced for at least 3 months postoperatively to improve sperm motility and vitality. ${ }^{9}$

\section{Study endpoint design and patency evaluation}

Follow-ups were completed via clinic visits or telephone contact. The presence of sperm in a semen sample at 3 months was used as the preliminary endpoint of this study. Postoperative semen analysis was performed at least once 3 months postoperatively in accordance with World Health Organization guidelines. Patency was defined as the



Figure 1 Placement of sutures in the single-armed LIVE technique (diagram). LIVE, longitudinal intussusception vasoepididymostomy. presence of sperm $\left(>10^{4} \mathrm{ml}^{-1}\right)$ in the semen sample. Complications were noted at each follow-up visit or contact.

\section{RESULTS}

Our modified single-armed LIVE technique was performed on 17 patients with EOA. Each patient provided at least one postoperative semen sample for analysis at 3 months (Table 1). Motile sperm were found in the bilateral epididymal fluid samples of 16 patients who underwent bilateral modified procedures. Of these 16 patients, two underwent repeated VEs with both anastomotic sites at the corpus after previously failed VEs. Those procedures were performed 40 and 36 months prior to the study. The patient who underwent unilateral VE had an occluded abdominal vasal segment on the left side, which was detected $18 \mathrm{~cm}$ from the vasal hemisected incision by a hydrophilic guidewire.

In this cohort, sperm $\left(>10^{4} \mathrm{ml}^{-1}\right)$ was present at 3 months postoperatively in the semen samples of $10(58.8 \%)$ patients, including one patient who underwent repeated VE. The median sperm density and forward motility were 12.2 million $\mathrm{ml}^{-1}$ and $0 \%-33 \%$, respectively. Normal sperm density $\left(>20\right.$ million $\mathrm{ml}^{-1}$ ) was found in four men. The patient who underwent unilateral VE failed to show sperm in his semen postoperatively.

The mean operative time from the start of the skin incision to the end of skin closure was $219 \mathrm{~min}$. No wound infections or sperm granulomas were observed in this study.

\section{DISCUSSION}

Microsurgical correction for EOA obviates the need for female ovarian stimulation and repeated sperm retrieval for each pregnancy. This procedure also improves the patient's self-image and allows for the natural selection of the best sperm. ${ }^{10,11}$ Moreover, it is a more costeffective management option.

Although obstructive azoospermia is one of the few clearly correctable causes of male infertility, microsurgery is a technically challenging procedure. The intussusception technique described by Berger ${ }^{4}$ in 1998 is an important step that simplifies the procedure, and it has been further modified. Marmar's modification ${ }^{5}$ of this technique 2 years later decreased the number of sutures from three to two and achieved a better patency rate than the previously described methods. Chan et al. ${ }^{6}$ then discontinued the use of a transverse suture placement and adopted a longitudinal placement in the epididymal tubule, which resulted in superior patency in rats. The longitudinal style allows a

Table 1 Preoperative variables and the postoperative outcomes of the patients

\begin{tabular}{lc}
\hline Items & Value \\
\hline Mean age (year) & 30.4 \\
Mean months of infertility & 38.4 \\
Surgery & 2 \\
$\quad$ Repeated & 16 \\
$\quad$ Bilateral & 1 \\
$\quad$ Unilateral & \\
Anastomotic site & 15 \\
$\quad$ Caudal & 2 \\
$\quad$ Corpus & 219 \\
Mean operative time (min) & 244 \\
$\quad$ First eight vasoepididymostomies (min) & 198 \\
$\quad$ Subsequent nine vasoepididymostomies (min) & $10 / 17(58.8 \%)$ \\
Overall patency rate & $12.2(1.0-30)$ \\
Mean sperm count (million ml $^{-1}$ ) & $0-33$ \\
Forward motility (\%) & \\
\hline
\end{tabular}


wider opening in the epididymal tubule, which increases the chances of patency initially and in the long term, and this finding is supported by some subsequent studies. ${ }^{2,3,11,12}$ These techniques usually use specialized double-armed sutures for the anastomosis, in which the needles are placed inside-out on the vas deferens to avoid back-walling the tubular lumen. However, these double-armed sutures are expensive and are difficult to acquire in some countries. In a study by Monoski et al., ${ }^{7}$ a novel single-armed suture technique was described and proved to be effective in a rat model. The authors suggested that if a single-armed suture technique could be performed successfully in rats, then it could naturally be used safely in humans as well. In 2011, we modified this single-armed technique and applied it to humans. Here, we present our modified technique with reasonable preliminary outcomes in 17 men with EOA. To our knowledge, this is the first investigation in humans that evaluated the effectiveness of applying a single-armed suture technique to perform an anastomosis between the vas deferens and epididymal tubule.

The advantage of using the double-armed suture is that it decreases back-walling of the vasal lumen due to the needles puncturing the lumen in an inside-to-outside fashion. However, using the singlearmed suture pattern, we dilated the vasal lumen to make it wide enough to pass the needle through the lumen with the aid of microforceps or a microneedle holder. The needle was then inserted through the vasal wall carefully in an outside-in fashion, which also avoided the needle hooking into the back wall of the lumen.

We modified Monoski's technique ${ }^{7}$ by passing the needle through the inferior points of the vasal mucosal layer in an outside-in fashion (a1 and b1; Figure 1), through the epididymal tubule, and finally through the superior points of the vasal mucosal layer in an insideout fashion ( $\mathrm{a} 2$ and $\mathrm{b} 2$; Figure 1). In our experience, this minor modification places the two sutures in a lower position when the needles are in the tubule, which can avoid crossing of the sutures. Moreover, the single-armed suture can be trimmed to an appropriate length of $4-5 \mathrm{~cm}$, which reduces crossing of the sutures and minimizes the manipulation of the delicate edges of the epididymal tubule when the sutures are pulled through to the end.

Monoski et al. ${ }^{7}$ did not show statistically significant differences in the patency rates between the single- and double-armed suture VE groups. Despite a short follow-up period, our study indicated a patency rate of $58.8 \%(10 / 17)$, which is comparable with the reported patency rate of $48 \%-85 \%$ for the double-armed suture VE technique. ${ }^{3,11,13}$ These data demonstrated that the modified single-armed suture technique accomplished the same effect as the double-armed suture approach, although the two were executed with different suture patterns.

In Kumar's study, ${ }^{13}$ the mean time to sperm positivity was only 3.2 months, with a maximum of 7 months. Thus, we expected most of the men to produce sperm by this time, but we still expected an improvement with further follow-ups. Another factor that may have an influence on the VE outcome is the aetiology of the epididymal obstruction. The cause of obstruction in most patients in this cohort is epididymitis. However, the patency rate in such patients has not been reported independently, so it is unclear whether inflammatory factors influenced the patency rate in this study. Additionally, one patient who received repeated VE using our modified technique also had sperm in his semen. Therefore, it may be worthwhile to offer such patients repeated VE after an unsuccessful procedure. ${ }^{14}$

The thickness of the needle may have contributed to these results. Initially, we used single-armed, 10-0 nylon sutures with a thicker needle. This size creates a larger hole in the epididymal tubule during the placement of the needles and often results in the partial collapse of the tubule, increasing the difficulty of subsequent operations. Subsequently, the use of single-armed, 10-0 prolene sutures with a $200-\mu \mathrm{m}$ needle was introduced to this study. This needle creates a smaller hole in the epididymal tubule and is preferable to use this precise procedure, although the 10-0 prolene sutures are not as soft as the 10-0 nylon sutures. If nylon sutures could be swaged onto a $200-\mu \mathrm{m}$ single-armed needle, their use would be ideal for the subtle anastomosis between such small tubules in cases when specialized double-armed sutures are unavailable.

The mean operating time for our procedures was slightly longer than that described in studies by Chan et al. ${ }^{1}$ or Kumar et al. ${ }^{13}$ but comparable with that reported by Ho et al. ${ }^{15}$ Our first eight procedures took $244 \mathrm{~min}$, whereas the last nine procedures only took $194 \mathrm{~min}$. These data indicated that the initial procedures took longer. We speculate that as we gain additional surgical experience, the operative time will continue to decrease.

In Chan's prospective study, ${ }^{1}$ the patency rate at the first semen analysis at 1 month was $60 \%$, and Kumar's study ${ }^{13}$ also showed a mean time of 3.2 months for the return of sperm. Thus, we presumed that most of our patients would have sperm by this time, and we identified the presence of sperm at 3 months as a preliminary endpoint for this study to evaluate the initial effectiveness of our modified technique in men with EOA.

However, the short follow-up time is the reason why we did not report the pregnancy rate. Moreover, we should note the small number of cases and the lack of a control group in our study, which may bias the results and make it difficult to draw meaningful statistical conclusions. Even so, we believe that our modified single-armed suture technique is a practical and effective alternative to doublearmed suture technique that may decrease the crossing of sutures and reduce operation times. Additionally, clinical investigations that include more cases with longer-term follow-ups will be indispensable for further multifactorial analyses.

In summary, this modified technique showed a reasonable patency rate in patients with EOA. Men who are candidates for the reconstruction of their reproductive tract could therefore be offered this option.

\section{AUTHOR CONTRIBUTIONS}

LZ carried out the microsurgical procedure and drafted the manuscript. CHD designed the study and drafted the manuscript. XZS provided important intellectual advice and helped to revise the manuscript. YC, WWW and LY-Zhao participated in the acquisition and analysis of the data. LY-Zeng analysed the epididymal fluid and semen samples. XAT conceived of the study and carried out the microsurgical procedure.

\section{COMPETING FINANCIAL INTERESTS}

All authors declare that there are no competing financial interests.

\section{ACKNOWLEDGMENTS}

This study was supported by the National Natural Science Foundation of China (No. 81172432, No. 81070488 and No. 30901487), Guangdong Natural Science Foundation (No. 9151802904000002) and Guangdong Provincial Ministry of Cooperation Project (No. 2011B090400034).

Supplementary Information accompanies the paper on Asian Journal of Andrology's website (http://www.nature.com/aja).

1 Chan PT, Brandell RA, Goldstein M. Prospective analysis of outcomes after microsurgical intussusception vasoepididymostomy. BJU Int 2005; 96: 598-601. 
2 Kumar R, Mukherjee S, Gupta NP. Intussusception vasoepididymostomy with longitudinal suture placement for idiopathic obstructive azoospermia. J Urol 2010 ; 183: 1489-92.

3 Schiff J, Chan P, Li PS, Finkelberg S, Goldstein M. Outcome and late failures compared in 4 techniques of microsurgical vasoepididymostomy in 153 consecutive men. J Urol 2005; 174: 651-5.

4 Berger RE. Triangulation end-to-side vasoepididymostomy. J Urol 1998; 159: 1951-3.

5 Marmar JL. Modified vasoepididymostomy with simultaneous double needle placement, tubulotomy and tubular invagination. J Urol 2000; 163: 483-6.

6 Chan PT, Li PS, Goldstein M. Microsurgical vasoepididymostomy: a prospective randomized study of 3 intussusception techniques in rats. J Urol 2003; 169: 1924-9.

7 Monoski MA, Schiff J, Li PS, Chan PT, Goldstein M. Innovative single-armed suture technique for microsurgical vasoepididymostomy. Urology 2007; 69: 800-4.

8 Oates R. Evaluation of the azoospermic male. Asian J Androl 2012; 14: 82-7.

9 Song FW, Zhong WD. Clinical efficacy of Shengjing capsule on patients with oligoasthenospermia. Zhonghua Nan Ke Xue 2009; 15: 762-4.
10 Lopushnyan NA, Walsh TJ. Surgical techniques for the management of male infertility. Asian J Androl 2012; 14: 94-102.

11 Peng J, Yuan Y, Zhang Z, Gao B, Song W et al. Patency rates of microsurgical vasoepididymostomy for patients with idiopathic obstructive azoospermia: a prospective analysis of factors associated with patency-single-center experience. Urology 2012; 79: 119-22.

12 Zhang GX, Bai WJ, Xu KX, Wang XF, Zhu JC. Clinical observation of loupe-assisted intussusception vasoepididymostomy in the treatment of obstructive azoospermia (analysis of 49 case reports). Asian J Androl 2009; 11: 193-9.

13 Kumar R, Gautam G, Gupta NP. Early patency rates after the two-suture invagination technique of vaso-epididymal anastomosis for idiopathic obstruction. BJU Int 2006; 97: 575-7.

14 Pasqualotto FF, Agarwal A, Srivastava M, Nelson DR, Thomas AJ Jr. Fertility outcome after repeat vasoepididymostomy. J Urol 1999; 162: 1626-8.

15 Ho KL, Wong MH, Tam PC. Microsurgical vasoepididymostomy for obstructive azoospermia. Hong Kong Med J 2009; 15: 452-7. 